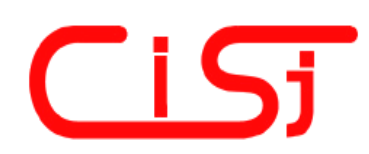

\title{
FUZZY QUERIES IN BLACK-SCHOLES MODEL FOR FINANCIAL OPTIONS
}

\author{
Arkadiusz Banasik \\ Silesian University of Technology, \\ Faculty of Organization and Management, \\ Department of Computer Science and Econometrics, \\ F. D. Roosevelt 26-28, 41-800 Zabrze, Poland, \\ arkadiusz.banasik@polsl.pl,http://www.roz6.polsl.pl/abanasik
}

\begin{abstract}
An approach how to use fuzzy queries in field of financial options is described. It provides the key aspect of information for investor expressed in natural language or queries in natural language. Presented paper indicates the first step in knowledge base creation for investor showing how to apply the corresponding mathematical apparatus to cope with natural language statements in Black-Scholes Model for option pricing.
\end{abstract}

Keywords: fuzzy logic, Black-Scholes Model, financial options, investment, artificial intelligence.

\section{INTRODUCTION}

Application of Computational Intelligence expanded rapidly during last years, and one of the most developing application areas is the financial world [1].

The modeling and trading of finances is a great challenge because of the huge number of factors involved in financial processes. These factors are among others: interest rates, exchange rates, the rate of economic growth, liquidity [1]. It occurs that 8.5 billion of shares are being traded daily in United States. That great number of shares is worth 120 billion dollars [1] showing its financial aspect indeed.

It is hard to estimate the influence of factors having impact on the financial assets prices because the effects can be non-linear, time-lagged and nonstationary [1].

All significant features of economy are based on information and knowledge, and the obtaining and representation of knowledge are essential research areas in Artificial Intelligence [1]. There is very important thing to make all necessary improvements of reasoning based on uncertain or imprecise information, which is the most common in order to experts' knowledge in investment field. This factor should be improved during scientific researches and studies. A process of information transforming into knowledge is well known but the methods for that transformation differ from the way of reasoning. To obtain the knowledge from the information it is necessary to gather data, and then transform it into helpful information. After that part of the process it is advisable to analyze that information and use it with our experience to create the knowledge from it. Finally we have to apply the obtained knowledge and find a solution for problem solving. We should note the application methods differ from the problem source as well as from the techniques of information transformation into knowledge.

The one of the problematic points is to provide tools for representing meaning of the natural language. For this purpose it is necessary to cope with such statements as e.g.: The interest rate is low and it is dropping. That statement contains fuzzy predicates, fuzzy quantifiers and fuzzy probabilities [2]. This is the key point of natural language, which describes all peoples' behavior. Appropriate processes of gathering and transformation of information into knowledge and rules are important aspects of $\mathrm{AI}$ and its use in practical cases.

There are different kinds of information: qualitative information, quantitative information and information presented in natural language. In order to quantitative information we do not need any other apparatus than mathematical and statistical ones. This approach is based on experts' opinions often used in order to financial matters. The application of Computational Intelligence (CI) in finance can be used in several main fields. It can be described generally as optimization methods, model induction techniques, financial forecasting, risk assessment, 
portfolio optimization and many others.

One of the most developing financial assets is an option whose value depends on the value of other underlying variables, and this value is derived, or contingent on other values. Typical underlying assets are stocks, stock indices, foreign and commodities [9]. There are two kinds of options: European and American, and both of them may be put or call options. An European call option (with strike price $K$, maturity date $T$ on an asset with price $P$ ) gives the holder the right (but not obligation) at $T$ to buy one unit of asset at price $K$ from the writer of the option. The strike and maturity date are decided when the option is written [9].

An European put option gives the holder the right (but not obligation) to sell the asset at stroke price $K$ at $T$ (date) [9].

Option pricing is concerned with pricing a random future payoff today, and it's based on obtaining a price which buyer and seller agree to enter during transaction. Option pricing can be achieved under assumption that market is with no arbitrage. It means there are no opportunities to make money out of nothing, and if two portfolios (or more) have the same cash flows in future, they must have the same value or price today [9].

The option pricing area is concerned with using a model of option pricing. One of the most popular models is the Black-Scholes Model which was used as a base for a proposed approach described below.

\section{CONCEPT AND APPROACH}

Taking into account the Black-Scholes Model (BSM) has many important features and assumptions let's consider some details.

The main assumptions are connected with features which are needed to use that model. The first assumption for market is to be arbitrage free. A second one is that markets are frictionless and continuous. It means there are no transactions costs or differential taxes, and the trading takes place all the time (continuously), and assets are infinitely divisible, and an unlimited borrowing is available there, and borrowing and lending rates both are equal [9]. The riskless instantaneous interest rate is constant over time, and the riskless bond price at time $u[9]$

$$
B_{u}=e^{r u} .
$$

The dynamics for the price of the risky traded asset $P$ (which pays no dividends) [9]

$$
\frac{d P_{u}}{P_{u}}=\mu d u+\sigma d W_{u}
$$

where $\mu$ is the instantaneous expected rate of return on asset $P, \sigma$ is instantaneous volatility, both constants and $W$ is a standard Brownian motion or Wiener process [9].

These dynamics mean that the asset $\mathrm{P}$ is longnormally distributed, hence

$$
P_{T}=p \exp \left\{\sigma\left(W_{T}-W_{t}\right)+\left(\mu-\frac{1}{2} \sigma^{2}\right)(T-t)\right\}
$$

where $p=P_{t}$ the current asset price [9].

Those assumptions above are used to prepare a final formula based on Black-Scholes Model. That formula for the European call option $F^{C}$ at time $0 \leq t$ $\leq T$ [9] can be described as:

$$
F^{C}(t, p)=p N\left(d_{1}\right)-K e^{-r(T-t)} N\left(d_{2}\right)
$$

where $K$ is a strike, $r$ is the riskless interest rate, $T$ is a maturity, $N(x)$ is the cumulative distribution of the standard Normal distribution, and

$$
d_{1}=\frac{\ln (p / K)+\left(r+\frac{1}{2} \sigma^{2}\right)(T-t)}{\sigma \sqrt{T-t}}, d_{2}=d_{1}-\sigma \sqrt{T-t}
$$

The Black-Scholes formula for the price of a European put option (with the same parameters) is a following [9]

$$
F^{p}(t, p)=K e^{-r(T-t)} N\left(-d_{2}\right)-p N\left(-d_{1}\right)
$$

It is possible:

- to investigate the effect of each parameter on the call or put price, holding the others fixed, and

- to think of $r$ and $\sigma$ as model parameters, $K$ and $T$ coming from the call or put option contract specification, and $t$ and $p$ the current state [9].

Those parameters above are expressed in numbers, however sometimes it's described by experts using natural language for example like high, low, medium, etc. Meantime the use of natural language is a way to cope with experts' source of information for Black-Scholes Model. Taking into account a presented concept is based on such kind of source with imprecise data and queries within BSM it's necessary to use the fuzzy sets in this case. A fuzzy set is an object characterized by its membership function which:

- is assigned per each object in the set, and

- is ranging between zero and one, and

- is the grade of membership of this object for mentioned set [3]. 
That definition above allows to declare more adequately the object is within a range of the set or not, and to be more precise with degree of being in range. This is a useful feature for expressions in natural language, e.g. the price is around thirty dollars, etc. It is obvious: if we consider just sets (not fuzzy sets) it will be very hard to declare objects and their membership function.

It is necessary to determine the meaning of membership function [5]. The first possibility is to indicate similarity between object and the standard.

A second one is to fix a level of preferences. In this case the membership function is considered as a level of acceptance for the object in order to declared preferences.

And the third possibility is to determine it as a level of uncertainty. In this case the membership function is considered as a level of validity that variable $X$ will be equal to value $\mathrm{x}$.

The third case (possibility) is concerned with Theory of Possibility [4] and this is an alternative towards Theory of Probability. It is assumed that Theory of Possibility is related to fuzzy sets, and it's is managed by defining the possibility distribution as a fuzzy restriction which behaves as an elastic constraint on the values (possible to be assigned to a variable) [4]. The approach in this case is definitely more useful in order to imprecise statement in natural language. Because it's too hard to use the Theory of Probability in such imprecise statement as e.g. the interest rate is very high, it's rather a level of similarity than probability of high interest rate. We should stress this approach is an important postulate which is the ground for analyzing natural language information and imprecise (fuzzy) statements.

Membership function is a base for gathering objects in fuzzy sets, as well as for further building of rules and contributions, etc. Information and data itself should be gathered for specified purposes, and the most suitable tool for that purpose is database. Basically a relational database is created by relations containing records. For a single relation every record has the same structure (the same number of attributes combined in the same way) and it is defined by its name and its attributes names [10].

The way of using databases is to create a query which is needed to extract appropriate data from database. A query may be formed as question for database to list records with criteria which are put to the system. Those criteria usually are defined numerically; however it is possible to introduce in the presented approach an imprecision for both data and queries.

The imprecise queries are often used in natural language, e.g. the price of the option is high, and it causes the database has to provide appropriate results. Imprecision and uncertainty in the knowledge about attribute $(A)$ can be expressed by possibility over the domain $X\left(\Pi_{A}\right)$.

Whenever data stored is uncertain or vague, or when the query is vague itself, a match between a unit of data and the query cannot be determined with a complete sureness because exact values of both data and query are not known with the precision [10]. The way of solving that problem is to implement the most favorable case and the less favorable case, and those two cases are called Possibility and Necessity correspondingly [10].

Let's consider an object $s$ of a data base, and $A(s)$ is one of the attributes, and $Q$ is a unit of query dealing with the same attribute, and $Q$ must be viewed as a union of possible solutions for achieving match. The simple for of relation is equality, but it is possible to specify as well: much bigger than, around, not. These relations are described by membership function $\mu_{r}[10]$.

The above mentioned functions have to be described. In particular the possibility measure [11]

$$
\operatorname{Pos}(r(a) / A(s))=\operatorname{SupMin}\left(\mu_{(a)}(x), \prod_{A(s)}(x)\right)
$$

where the value of the attribute $A$ for the object $s$ is the set of elements that are in relation $r$ at least one element of $a$. And the necessity of the same event (the value of attribute $A$ for the object $s$ is the set of elements which are in relation $r$ with at least one element of a) can be described as the necessity measure [11]

$$
\operatorname{Nec}(r(a) / A(s))=\operatorname{InfMax}\left(\mu_{(a)}(x), 1-\prod_{A(s)}(x)\right)
$$

with

$$
\mu_{r(a)}(x)=\operatorname{SupMin}\left(\mu_{r(x, y)}, \mu_{a}(y)\right)
$$

where $\mu_{a}$ is the membership function indicating the atom, and $\mu_{r}$ is the membership function of relation which can be fuzzy, defined over the cross product $X x X$ where $X$ is the universe of discourse for the attribute $A$.

It should be noted that in case where the information stored in the data base $A(s)$ is precise the two matching degrees are equal, in other circumstances they are not.

\section{EXPERIMENT AND IMPLEMENTATION}

The experiment below shows the possibility of using natural language in values of input data to Black-Scholes Model and the following tables (Table 1 and Table 2) indicate the differences 
between numerical values and fuzzy values. Values in Table 1 are counted on the base of formulas $1-6$ and those formulas above can be used for further improvements. It allows changing input data from numerical into natural language statements, which is a main point of further reasoning.

Table 1. Numerical input data

\begin{tabular}{|c|c|c|c|c|c|}
\hline $\begin{array}{l}\mathscr{\Xi} \\
\text { Z }\end{array}$ & 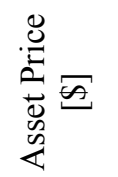 & 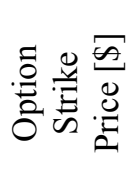 & 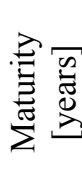 & 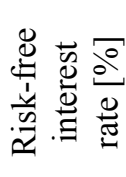 & 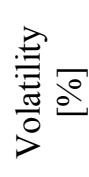 \\
\hline $\mathrm{A} 1$ & 25,00 & 15,00 & 3,5 & 3 & 20 \\
\hline $\mathrm{A} 2$ & 30,00 & 10,00 & 2 & 5 & 10 \\
\hline $\mathrm{A} 3$ & 40,00 & 50,00 & 1 & 7 & 25 \\
\hline A4 & 100,00 & 20,00 & 5 & 3 & 25 \\
\hline
\end{tabular}

As it can be seen from Table 2 there are expressions in natural language instead of numerical values. That transformation is carried out on base of equations 7 and 8 . This is a first approach connected with natural language using. It is often implemented in order to experts' statements, when they declare such features of assets as low, medium or high. That approach shows a possibility of natural language using in input data for databases. Connection between those two tables is based on membership function, which provides pieces of information allowing us to include numerical values to values expressed in natural language.

A second approach is dealing with imprecise queries in natural language as well as expressions such as: around, between, etc., for example maturity around two years.

Table 2. Natural language input data

\begin{tabular}{|c|c|c|c|c|c|}
\hline $\begin{array}{l}\text { Æ } \\
\text { Z } \\
\text { Z }\end{array}$ & 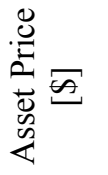 & 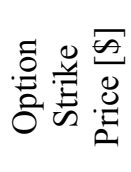 & 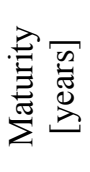 & 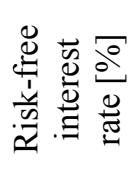 & $\stackrel{\substack{0 \\
\frac{\pi}{0}}}{\frac{0}{0}}$ \\
\hline A1 & 25,00 & 15,00 & 3,5 & Low & High \\
\hline A2 & 30,00 & 10,00 & 2 & Medium & Low \\
\hline A3 & 40,00 & 50,00 & 1 & High & High \\
\hline A4 & 100,00 & 20,00 & 5 & Low & High \\
\hline
\end{tabular}

The implementation code is made in Prolog, and possible queries are introduced below for presented tables. In particular the following queries

Table 1(A1, 25.00,15.00, around(3), 3, around(15)) Table 1(A2, unknown, unknown, 3.5, 3, 20)

illustrate the first approach containing imprecise queries in experts' statements. And queries
Table 2(A1, 25.00, 15.00, 3.5, Low, High)

Table 2(A2, 30.00, 10.00, 2, Medium, Low)

are using a natural language in process of query creation which allows us to ask the database in more natural way - more adequate for people.

That approach has to provide information for investor's knowledge base which is a main point of further research discovering the knowledge from imprecise data [11]. Presented algorithm (Fig. 1.) is the base of decision support system for investor, and its main feature is to cope with imprecise data and imprecise queries [11]. That algorithm starts from entering data connected with pieces of information collected by user and based on his or her knowledge about enterprises, which stocks are being investigated to buy (Block - 1). It causes the procedure to last as long as the user enters data completely (sometimes it makes him or her to do it till all data are entered to the system).

After the first part of all user information entering was completed a system allows to import the additional information from other sources (Block -2 ) and the enterprises are being analyzed in order to added data and user's preferences (Blocks -3 and $4)$.

Many pieces of information from user are declared in natural language so it is necessary to combine membership functions based on the information selected before (Block -5 ). Then the system creates a list of enterprises to be a part of the further analysis and the enterprises are being put in appropriate order(according to user's preferences) on the list (Blocks -6 and 7).

In process running the system asks for additional data from user to declare if the order on the list is correct. If the answer is "Yes" it generates the list of enterprises which are being chosen to be a part of option portfolio of that exact investor (Block -8).

The darkest blocks are presenting the key decisions in the running algorithm, so a proposed approach is a part of decision support system creation. A block Continue represents the necessity to gather more information as well as indicates a step back in the algorithm. It is the same block in all three situations repeating in the algorithm (see Fig.1).

It should be noted:

- Presented concept is a main part of implementation of the algorithm and uses PROLOG and natural language to provide better understanding of financial matters for the investor

- Proposed approach is the key point in creation and implementation of decision support system based on Black-Scholes Model for 
options' pricing

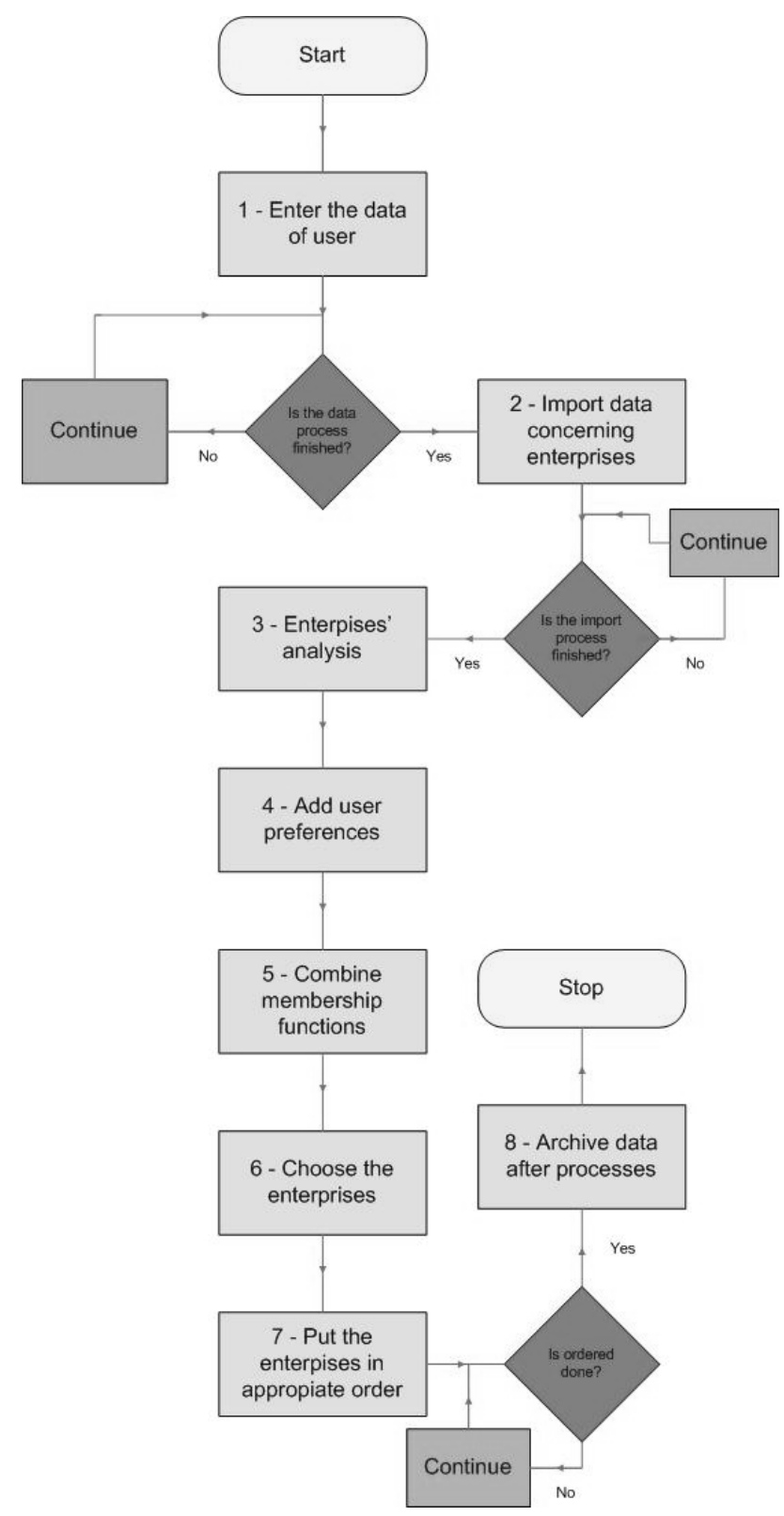

Fig. 1 - Knowledge discovery algorithm

\section{CONCLUSIONS AND FUTURE WORK DISCUSSION}

Presented paper is a starting point of creating a knowledge base for investor. It only demonstrates a possibility of imprecise and uncertain data storing and it's followed using in a way that is closer to commonsense understanding.

One of the paper outcomes is that once a data base is complete and every possible concept is expressed by fuzzy numbers it is no longer useful to remember what was the way of creation and storage of important data. That is a good feature for firsttime users.

To be more adequate it is a good point in presented solution that user can decide on his or her own whether he or she wants to be very strict and consider only relevant data or on the other hand allows only a slight match.

Creation of the useful and simple decision support system for the investor can be a key point in future works. That approach leads to implementation of the natural language use in fields of data and reasoning.

The proposed fuzzy based approach is a promising direction for further researches especially in such specific areas of interests as finance and stock exchange.

\section{REFERENCES}

Algos 3.0. Developments in Algorithmic Trading. Traders Magazine 2007. Special Report. Source Media's Custom Publishing Group.

[2] A. Brabazon. M. O'Neill. I. Dempsey. An Introduction to Evolutionary Computation in Finance. IEEE Computational Intelligence, Vol. 3 No. 4 (November 2008), pp. 42-55.

[3] L. Zadeh. Knowledge Representation in Fuzzy Logic. IEEE Transactions on Knowledge and Data Reasoning, Vol. 1 No. 1 (March 1989), pp. 89-100.

[4] L. Zadeh. Fuzzy Sets. Information and Control (8) (1965), pp. 338-353.

[5] L. Zadeh. Fuzy Sets as a Basis for Theory of Possibility. Fuzzy Sets and Systems 1 (1978), pp. 3-28.

[6] J. Łęski. Systemy Neuronowo-rozmyte. WNT. Warszawa, 2008. p. 689.

[7] J. Chojcan, J. Łęski. [Eds.] Zbiory Rozmyte $i$ Ich Zastosowania. Wydawnictwo Politechniki Śląskiej. Gliwice, 2001. p. 479.

[8] D. Dubois. H. Prade. Fuzzy Sets in Approximate Reasoning, Part 1: Inference with Possibility Distributions. Fuzzy Sets and Systems 40 (1991), pp. 143-202.

[9] D. Dubois. H. Prade. The Three Semantics of Fuzzy Sets. Fuzzy Sets and Systems 90 (1997), pp. 141-150.

[10] V. Henderson. The Black-Scholes Model. Lectures University of Oxford. February 19, 2003, p. 22.

[11] M. Uszynski. Fuzzy queries with linguistic quantifiers for information retrieval from data bases. Technical report CSD-87-333. University of California. Berkeley, 1980, p. 11.

[12] A. Banasik. Knowledge representation and reasoning for investor. Systemy Wspomagania Organizacji SWO 2008. [Eds.] M. Pańkowska, T. Porębska-Miąc, H. Sroka. Wydawnictwo Akademii Ekonomicznej im. Karola 
Adamieckiego w Katowicach, Katowice 2008, pp. 373-381.

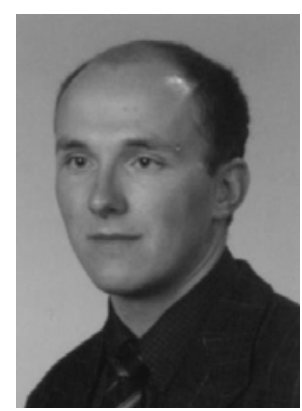

Ошибка! Недопустимый

объект гиперссылки. (MSc.

Eng. 2004 Silesian University of

Technology), was born in

Zabrze in 1980, assistant on

Silesian University of

Technology. Scientific areas of

interests: Fuzzy Logic, Neural

Networks, Al. Actual field of

researches is connected with

implementation of Al methods in finance. That area

of interest is connected with PhD Thesis supervised

by prof. Jacek Łęski. During his scientific career he

is a member of Information Technology Group on

Silesilesian University of Technology, he is a member of IEEE and IEEE Computational Intelligence Society, member of ACM and member of Polish Information Processing Society. 